\title{
Thermodynamic Model of Mars Oxygen ISRU Experiment (MOXIE)
}

\author{
IAC-15.B3.7.10 \\ Forrest E. Meyen ${ }^{1}$, Michael H. Hecht ${ }^{2}$, Jeffrey A. Hoffman ${ }^{3}$ and the MOXIE Team
}

\begin{abstract}
As humankind expands its footprint in the solar system, it is increasingly important to make use of the resources already in our solar system to make these missions economically feasible and sustainable. In-Situ Resource Utilization (ISRU), the science of using resources at a destination to support exploration missions, unlocks potential destinations by significantly reducing the amount of resources that need to be launched from Earth. Carbon dioxide is an example of an in-situ resource that comprises $96 \%$ of the Martian atmosphere and can be used as a source of oxygen for propellant and life support systems. The Mars Oxygen ISRU Experiment (MOXIE) is a payload being developed for NASA's upcoming Mars 2020 rover. MOXIE will produce oxygen from the Martian atmosphere using solid oxide electrolysis (SOXE). MOXIE is on the order of magnitude of a $1 \%$ scale model of an oxygen processing plant that might enable a human expedition to Mars in the 2030's through the production of the oxygen needed for the propellant of a Mars ascent vehicle. MOXIE is essentially an energy conversion system that draws energy from the Mars 2020 rover's radioisotope thermoelectric generator and ultimately converts it to stored energy in oxygen and carbon monoxide molecules. A thermodynamic model of this novel system is used to understand this process in order to derive operating parameters for the experiment. This paper specifically describes the model of the SOXE component. Assumptions and idealizations are addressed, including 1D and 2D simplifications. Operating points are discussed as well as impacts of flow rates and production.
\end{abstract}

Index Terms-MOXIE, ISRU, Solid Oxide Electrolysis, Mars 2020

\section{INTRODUCTION}

A paradigm shift in space exploration has begun. Space explorers have always been limited by what they could bring with them. Now, technologies for In-Situ Resource Utilization (ISRU), the art of "living off the land", are paving the way for a sustainable human presence in space. Carbon dioxide is an example of an in-situ resource that comprises most of the Martian atmosphere and can be used as a source of oxygen for propellant and life support systems. The Mars Oxygen ISRU Experiment (MOXIE) will unlock this resource using a process called solid oxide electrolysis as an experimental payload aboard NASA's upcoming Mars 2020 rover.

\section{BACKGROUND}

\section{A. Motivation for ISRU}

The most recent NASA Design Reference Mission, DRM 5 Drake (2009), specifically calls for the development of ISRU technology to return astronauts from the surface of Mars by a Mars Ascent Vehicle. The system described would be an atmospheric ISRU plant that produces $\mathrm{O}_{2}$ from the $\mathrm{CO}_{2}$ in the atmosphere using solid oxide electrolysis. Additional processing steps could also generate the buffer gasses such as $\mathrm{N}_{2}$ and Ar that are also present in the atmosphere. The ISRU system would be sent ahead of the crew and the oxidizer tanks confirmed full before the crew would commit to the mission. Advantages of such a system include reducing total mass, reduced lander size and volume, improved surface exploration capability, life support redundancy, reduced mission risk from less launches, and lower life cycle costs.

One of the major disadvantages of such a system is that without extra fuel during landing, abort to orbit (ATO is not an option). NASA DRM 5 addressed this concern after concluding that abort to orbit is not feasible in any case during the most critical parts of landing, so the focus should be on abort to surface landing accuracy.

The primary goal of MOXIE is to be a pathfinder mission for future similar ISRU technologies. The ability to use oxygen already on Mars, freely available in the atmosphere is a game changing technology. It will enable a whole new class of sustainable missions by reducing the amount of material that needs to be brought on a mission. The potential uses for this oxygen production technology include

1. Producing oxidizer for a return trip from Mars

2. Generating oxygen for life support systems

3. Scrubbing CO2 from life support systems

Application 1 is the primary motivator for MOXIE, as the propellant costs to return from Mars can be extraordinary. It has been estimated that $29.3 \mathrm{mT}$ of oxygen will need to be produced to return astronauts from the Mars surface to a staging orbit around Mars. ISRU can harvest this oxygen already on Mars, resulting in a $29 \%$ reduction in landed mass, effectively reducing the initial mass required in Low Earth Orbit (LEO) by $336 \mathrm{mT}$. (Rapp, Hoffman et al. 2015). Integrating a full scale MOXIE like system into future designs would save nearly 3 SLS launches at their designed capacity of 130 metric tons (Senate" 2010). While the final launch costs are uncertain, at \$0.5-5 billion per launch (Strickland 2013), an ISRU system could potentially save tens of billions of dollars.

\section{B. System Overview}

The MOXIE system is designed to be a miniature oxygen production plant on Mars. It will produce oxygen at a rate that is on the order of magnitude of a $1 \%$ scale model of an ISRU system required to support oxidizer fueling for a Mars mission. MOXIE consists of three major subsystems.

1. Carbon dioxide Acquisition and Compression (CAC)

2. Solid Oxide Electrolysis (SOXE)

3. Monitor and Control System (MCS) 
This paper focuses on describing models for system 2, the SOXE. Here we will briefly describe the other sub-systems. A simplified depiction of the MOXIE subsystems is shown in Figure 1.

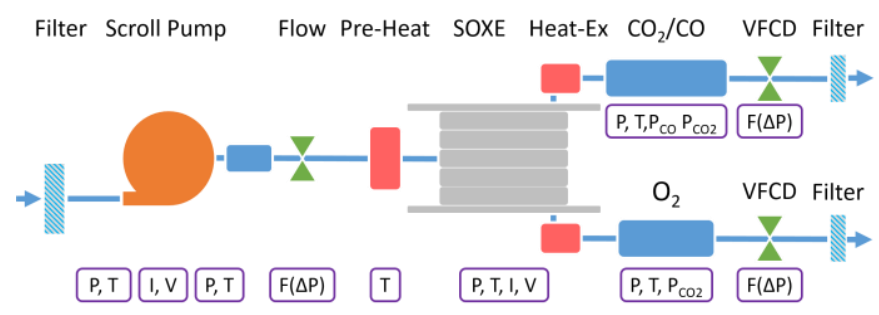

Figure 1 - Outline of MOXIE subsystems

\section{Carbon Dioxide Acquisition and Compression}

Martian atmosphere is drawn through a dust filter by the MOXIE scroll pump (Figure 2), a mechanical pump that volumetrically compresses the atmosphere to 1 Earth atmosphere at a compression ratio on the order of $100 \mathrm{x}$. There is currently some uncertainty in the landing site location of the Mars 2020 rover. Atmospheric pressure of Mars varies by altitude and season, with an average pressure of $636 \mathrm{~Pa}$ at mean radius (Williams 2015). Lower inlet pressures increase the energy required to deliver compressed $\mathrm{CO}_{2}$ to the SOXE. Reduction of the inlet pressure also affects the pump performance as a result of a scroll pump characteristic that is a lower volumetric efficiency resulting from larger delta P.

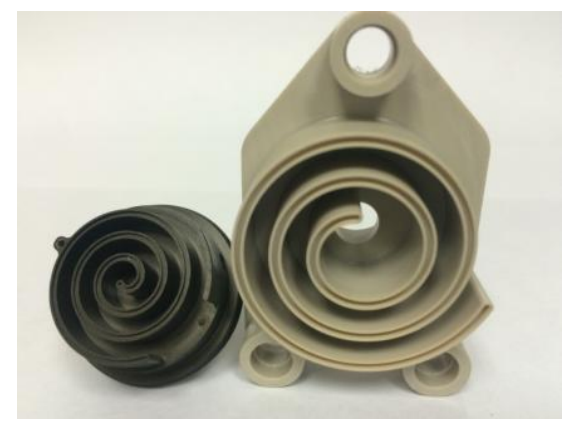

Figure 2 - A scroll pump consists of a fixed and rotating set of involutes. PTFE seals at the face of each scroll help seal the pump and reduce leaking, which could decrease volumetric efficiency.

After the scroll pump there is a small volume, or "plenum" to help buffer the SOXE from any pressure spikes from the rotating pump. Characterization of the pump design will improve our models of pump volumetric efficiency for different pressure ratios. A key advantage of these types of pumps is that they enable a one-step system with on-demand pressure. A challenge with this type of pump is that, over time the tip seals may wear and reduce volumetric efficiency.

A secondary option for the CAC system was to use cryopumps that would freeze $\mathrm{CO}_{2}$ out of the Martian atmosphere. This system required two steps, an acquisition period where dry ice is collected, and a pressure stage where the dry ice is sublimated to generate pressure to feed into the SOXE. This method has lower peak power than the scroll pump method but uses more power per collection cycle(Rapp, Hoffman et al. 2015).

\section{SOXE cells and stack configurations}

The oxygen generating chemical reaction takes place inside the solid oxygen electrolysis (SOXE) stack. The base unit of the SOXE is a cell. Each cell contains an oxygen permeable electrolyte, sandwiched between a cathode and an anode. Atmospheric gas flows over the cathode where the reduction reaction takes place. Oxygen ions transport through the electrolyte and form $\mathrm{O}_{2}$ in the anode. Multiple SOXE cells in a SOXE "stack" configuration. A stack is a group of cells connected through interconnects that guide gas flow across the cells. The cells in the stack are connected in series and a voltage is applied across the stack. The current through each cell in the stack is the same, but the voltage drop varies across each cell depending on the electrochemical resistance of that cell. As elaborated later, all the current is ionically transmitted through each cell so the production rate of each cell in a stack is the same. Figure 3 shows a SOXE system with two stacks of 11 cells each.

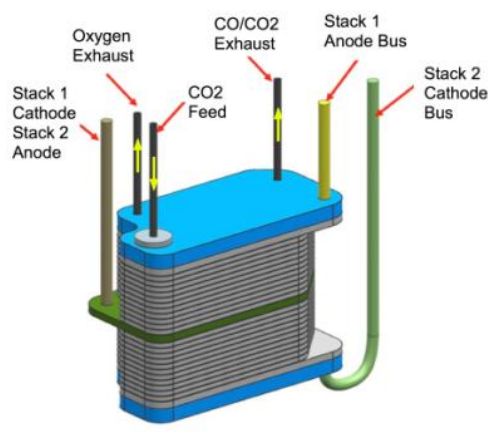

Figure 3 - Two SOXE Stacks. A center voltage tab (green) separates the two stacks.

\section{E. Monitoring and Control System}

The monitoring and control system (MCS) characterizes the states of the inlet gas, pump health, state of the electrochemical process, and the outlet gas composition. Figure 1 indicates the system sensors and locations outlined in purple. Pressure and temperature measurements are taken at the exit of the inlet filter to characterize the gas stream entering the scroll pump. The SOXE inlet gas flow rate is characterized by an orifice plate calibrated for a pressure drop, flow rate, relationship. The controlled processes include

1. The scroll pump speed

2. SOXE inlet gas heater temperature

3. SOXE temperature

4. SOXE voltage

5. Outlet gas analysis system temperature

Feedback is incorporated to prevent hazardous system states. An elaborated description of the control system can be found in (Rapp, Hoffman et al. 2015). 


\section{Modeling SOlid OXIDE Electrolysis}

\section{A. MOXIE Primary Chemical Reaction.}

MOXIE produces $\mathrm{O}_{2}$ through the process of high temperature $\mathrm{CO}_{2}$ electrolysis. The net chemical reaction is

$$
2 \mathrm{CO}_{2} \rightarrow 2 \mathrm{CO}+\mathrm{O}_{2}
$$

The reaction occurs in a solid oxide electrolysis cell (SOEC). The SOEC cell and supporting system is referred to as the SOXE subsystem of the MOXIE instrument. A SOXE cell consists of a porous anode and porous cathode, separated by a zirconia ceramic electrolyte. The cathode, includes catalytic sites that promote the reaction

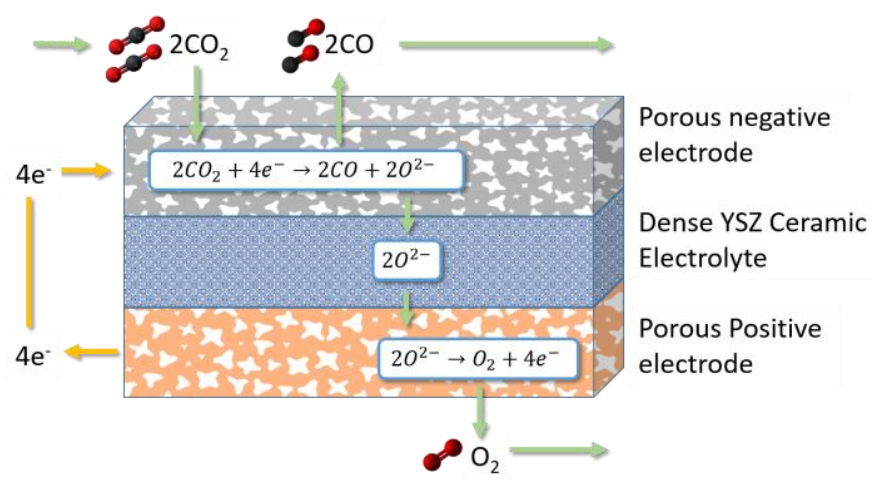

Figure 4 - Reactions across a SOXE cell

As the $\mathrm{CO}_{2}$ flows through the porous cathode, the $\mathrm{CO}_{2}$ comes in contact with catalytic sites while an electronic potential is applied. The $\mathrm{CO}_{2}$ is reduced to $\mathrm{CO}$ and a negatively charged $\mathrm{O}^{2-}$ atom.

$$
2 \mathrm{CO}_{2}+4 e^{-} \rightarrow 2 \mathrm{CO}+2 \mathrm{O}^{2-}
$$

The potential drives the charged $\mathrm{O}^{2-}$ ions through the yttriastabilized zirconia (YSZ) electrolyte. Once in the positive electrode material, the two $\mathrm{O}^{2-}$ ions are oxidized per $\mathrm{O}_{2}$ molecule formed.

$$
20^{2-} \rightarrow \mathrm{O}_{2}+4 e^{-}
$$

The YSZ electrolyte is ionically but not electrically conductive. The current efficiency or Faraday efficiency $\left(\eta_{F}\right)$ is the ratio of ionic current transfer over total current transfer (Nehrir and Wang 2009) and can be described by the following relation.

$$
\eta_{F}=\frac{I_{\text {ion }}}{I_{\text {tot }}}=1-\frac{I_{\text {para }}}{I_{\text {tot }}}
$$

Where $I_{i o n}$ is the ionic current, $I_{\text {para }}$ is the parasitic, or electronic current and $I_{\text {tot }}$ is the total current transfer. Experiments using YSZ electrolyte for solid oxide electrolysis of $\mathrm{CO}_{2}$ have shown that practically all current is ionically transferred (Sridhar and Vaniman 1997). Therefore, we can assume that for the SOXE:

$$
\eta_{F}=1
$$

This property of the YSZ electrolyte used in the SOXE enables MOXIE to perform real time monitoring of oxygen production rates. Because all electrons are ionically transferred with an oxygen ion, and four electrons are transferred per mole of $\mathrm{O}_{2}$, the molar production rate can be calculated from the stack current measurement.

$$
\dot{n}_{O_{2}}\left[\frac{m o l}{s}\right]=\frac{I}{4 F}
$$

Where $\dot{n}_{\mathrm{O}_{2}}$ is the molar flow rate of oxygen in moles per second, $I$ is the stack current, and $F$ is Faraday's constant, 96,485 Coulombs per mole of electrons. MOXIE $\mathrm{O}_{2}$ production is measured on the order of grams per hour. Converting time and the molar mass of oxygen yields

$$
\dot{m}_{O_{2}}\left[\frac{g}{h r}\right]=\frac{I}{3.3503}
$$

This is the relationship between measured current and oxygen production for a single cell in the SOXE stack. In the configuration for MOXIE, all cells are connected in series, and each cell has the same current. Production rate in each cell in a stack is the same, while voltages may vary per cell depending on differences in production.

The YSZ electrolyte must also be hot to permit oxygen ion transport. The MOXIE design reference temperature for the SOXE is $1073 \mathrm{~K}$. The resistance of the electrolyte to oxygen ion transport decreases at higher operating temperatures, a design parameter that must be balanced with the energy required to warm the stack before operation, and the acceptable heat load of the payload and rover. The heat consumption and production of the electrochemical reactions must also be weighed when determining the operating temperature and voltage.

\section{B. Relating Flow Rate to Utilization Rate.}

Because we have a good measurement of current, which is also a direct correlation to $\mathrm{O}_{2}$ production, we can calculate the utilization rate from flow rate and stoichiometry. Using the reaction in Equation (1) and rewriting the balance with molecular weights of the species we have

$$
2\left(44\left[\frac{\mathrm{g}}{\mathrm{mol}}\right]\right)=2\left(28\left[\frac{\mathrm{g}}{\mathrm{mol}}\right]\right)+32\left[\frac{\mathrm{g}}{\mathrm{mol}}\right]
$$

So the ratio of $\mathrm{CO}_{2}$ consumed per gram of $\mathrm{O}_{2}$ produced is

$$
\frac{88}{32}=2.75 g_{C O_{2}} \text { cons.per } g \mathrm{O}_{2} \text { gen } \text {. }
$$

So the utilization rate can be calculated by

$$
\mathrm{CO}_{2} \text { Util. Rate }=\frac{\dot{m}_{\mathrm{CO}_{2 \text { cons }}}}{\dot{m}_{\mathrm{CO}_{2 \text { in }}}}=\frac{\left(2.75 * \dot{m}_{\mathrm{O}_{\text {out }}}\right)}{\dot{m}_{\mathrm{CO}_{2 \text { in }}}}
$$

$\mathrm{CO}_{2}$ utilization rate is an important parameter for monitoring the MOXIE system. Low utilization rates signal that the system flow rate is in excess of what the cell is able to 
process. In the event of low $\mathrm{CO}_{2}$ utilization, energy is being wasted by compressing excess $\mathrm{CO}_{2}$ in the acquisition process. If the utilization rate is high, it signals that the system is a higher risk for degradation processes such as carbon deposition on the SOXE cathode.

\section{Parametrizing Complex Electrochemical Dynamics to Area Specific Resistance.}

Area specific resistance (ASR) is simple to derive experimentally but much more complex to model and predict before testing. Factors that influence it include electrode kinetics, gas diffusion, and local variations in flow, temperature, and current density. By embodying these terms into ASR we reduce key parameters down to a variation of Ohm's law. The units are typically ohms ${ }^{*} \mathrm{~cm}^{2}$. ASR is described this way in (Ebbesen and Mogensen 2009) and is used to help quantify performance in testing data.

$$
A S R=\frac{\left(V_{o p}-V_{o c v}\right)}{i_{d e n}}
$$

Where $V_{o p}$ is the operating voltage and $V_{o c v}$ is the open circuit voltage, also known as the Nernst potential, and $i_{d e n}$ is the current density in amps per $\mathrm{cm}^{2}$. The average area of each cell in MOXIE is near $21 \mathrm{~cm}^{2}$

$$
A S R=\frac{\left(V_{o p}-V_{o c v}\right) / N_{c e l l}}{i_{d e n}}
$$

Where $N_{\text {cell }}$ is the number of equally sized cells in the stack.

One of the major challenges of $\mathrm{CO} 2$ electrolysis of the Martian atmosphere for the MOXIE experiment is that the electrolysis process is done without the addition of additional moisture. The reaction in the presence of water vapor is called co-electrolysis. In the presence of water, an additional reaction can take place that will enable the production of syngas, which improves efficiency and could be used for fuel production.

$$
\mathrm{H}_{2} \mathrm{O}+\mathrm{CO}_{2} \rightarrow \mathrm{H}_{2}+\mathrm{CO}+\mathrm{O}_{2}
$$

This reaction is much more favorable and decreases ASR. Results from SOEC stacks in an Idaho National Lab study published by Stoots et al. show dry $\mathrm{CO}_{2}$ electrolysis with an approximate ASR of $3.84 \Omega \mathrm{cm}^{2}$ while results for $\mathrm{H}_{2} \mathrm{O}$ coelectrolysis were around $1.38 \Omega \mathrm{cm}^{2}$ (Stoots, O'Brien et al. 2008) This improvement demonstrates great promise for solid oxide electrolysis ISRU system on Mars that can leverage both the local water and ambient carbon dioxide. For the purpose of this paper and modeling discussion, we will assume an ASR from the system most similar to MOXIE, and estimate our peak dry electrolysis ASR to be similar to the value measured in (Stoots, O'Brien et al. 2008), approximately $4 \Omega \mathrm{cm}^{2}$.

ASR for SOECs with YSZ electrolytes generally improves (lower resistance) as operating temperature increases. For example, YSZ electrolyte doped with 8 mol- $\%$ yttria $\left(\mathrm{Y}_{2} \mathrm{O}_{3}\right)$ has an ionic conductivity of $0.1 \mathrm{~S} / \mathrm{cm}$ at $1273 \mathrm{~K}\left(1,000{ }^{\circ} \mathrm{C}\right)$ but falls to $0.03 \mathrm{~S} / \mathrm{cm}$ at $1073 \mathrm{~K}\left(800{ }^{\circ} \mathrm{C}\right.$ ) (Bove and Ubertini 2008). This initial model assumes a constant temperature and a constant ASR. The MOXIE team is currently characterizing stack configurations. From this data an enhanced model will be derived for how ASR varies with temperature and other test conditions.

\section{Thermal Neutral Voltage}

The thermal neutral voltage is the voltage where all energy necessary for the reaction is provided electrically. At this point the reaction is neither endothermic nor exothermic. The voltages exceeding the thermal neutral voltage result in a net exothermic reaction. The thermal neutral voltage can be calculated by

$$
V_{t n}=\frac{\Delta H_{f}}{4 F}
$$

Where $\Delta H_{f}$ is the enthalpy of reaction and $4 F$ is the charge transferred in the reaction as written in Equation (1). In a temperature range from $800 \mathrm{~K}$ to $1400 \mathrm{~K}$ the thermal neutral voltage can be calculated by second order polynomial fit:

$$
\begin{gathered}
V_{t n}=-8.655 * 10^{-9}\left(T^{2}\right) \\
\ldots-1.496 * 10^{-6} *(T)+1.475
\end{gathered}
$$

Evaluating at the SOXE reference operating temperature $1073 \mathrm{~K}(800 \mathrm{C}), V_{t n}=1.463 \mathrm{~V}$.

\section{E. The Open Circuit Voltage or Nernst Potential}

The Nernst potential is represented in (Hartvigsen, Elangovan et al. 2015), and (Ni 2010) as

$$
V_{o c v}=E=E_{0}+\frac{R T}{2 F} \ln \left[\frac{P_{C O}^{0}\left(P_{O_{2}}^{0}\right)^{\frac{1}{2}}}{P_{C O_{2}}^{0}}\right]
$$

Where $P_{\mathrm{CO}}^{0}$ and $P_{\mathrm{CO}_{2}}^{0}$ are the partial pressures of $\mathrm{CO}$ and $\mathrm{CO}_{2}$ at the cathode, and $P_{O_{2}}^{0}$ is the partial pressure of $\mathrm{O}_{2}$ at the anode. $\mathrm{R}$ is the universal gas constant, $\mathrm{T}$ is the operating temperature and F is Faraday's constant. The factor 2 is the number of electrons transferred per oxygen atom. $\mathrm{E}_{0}$ is the equilibrium potential (or free energy potential) at standard pressure. (Hartvigsen, Elangovan et al. 2015) For consistency it has been rewritten here for the chemical balance described in Equation (1).

$$
V_{o c v}=V_{\text {rev }}+\frac{R T}{4 F} \ln \left[\frac{\left(P_{C O}^{0}\right)^{2} P_{O_{2}}^{0}}{\left(P_{C O_{2}}^{0}\right)^{2}}\right]
$$

Where the reversible voltage $V_{\text {rev }}$ can be calculated from thermodynamic tables and evaluated at any operating temperature.

$$
V_{\text {rev }}=\frac{-\Delta G_{f}^{\circ}}{4 F}
$$

Where $\Delta G_{f}^{\circ}$ is the standard free energy of reaction. $V_{r e v}$ can be linearly approximated over a temperature range from $800 \mathrm{~K}$ to $1400 \mathrm{~K}$ : 


$$
V_{\text {rev }}=-4.4924 * 10^{-4}(T)+1.4629
$$

At the reference operating temperature of $1073 \mathrm{~K}$, it can be evaluated that $V_{\text {rev }}=0.9808 \mathrm{~V}$. These equations can model open circuit voltage as a function of molar composition. This is important because the molar composition changes as the fluid flows across the stack and a portion of the $\mathrm{CO}_{2}$ is reduced. Figure 5 displays the results of this model for the Nernst potential as a function of molar fraction $\mathrm{CO} 2$ to $\mathrm{CO}$ and temperature. The pressure of $\mathrm{O}_{2}$ effluent on the anode is assumed to be 1 atmosphere as controlled by restrictions on gas flow exiting MOXIE. The incoming gasses are assumed to be pressurized to 1 atmosphere by the MOXIE scroll pump.

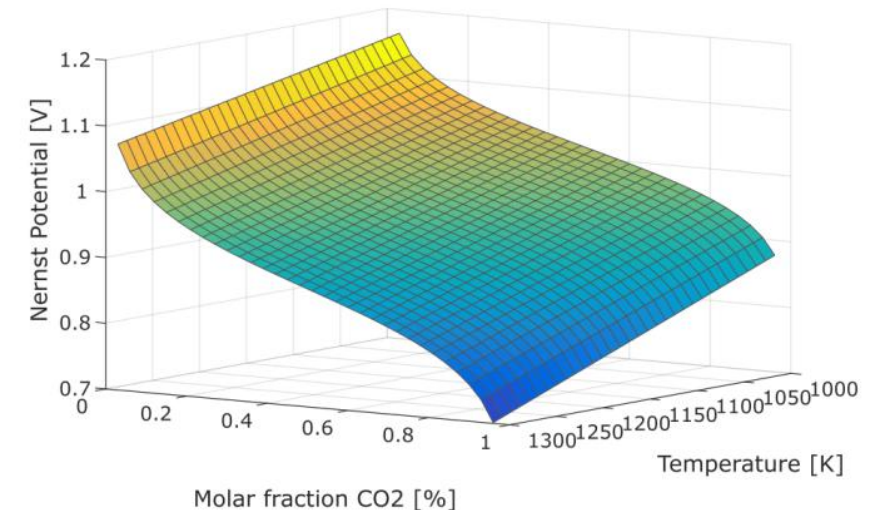

Figure 5 - The Nernst potential as a function of molar fraction $\mathrm{CO}_{2}$ to $\mathrm{CO}$ and temperature.

Figure 5 depicts the Nernst potential as a function of molar fraction of $\mathrm{CO}_{2}$ with respect to $\mathrm{CO}$, and the operating temperature. Equation (11) demonstrates that lower Nernst potentials result in higher current, and thus oxygen production rates for a given ASR. General trends are that the Nernst potential decreases with higher temperature and with higher concentration of $\mathrm{CO}_{2}$ with respect to $\mathrm{CO}$.

\section{F. $2 D$ approximation of Nernst Potential}

The reactant gas composition flowing over the catalytic sites of the cathode changes as the gas flows longitudinally through the cathode. At the entrance to the cell, the gas is the composition of the Martian atmosphere, approximately 95\% $\mathrm{CO}_{2}$ with only trace amounts of $\mathrm{CO}$. Alternative stack designs that include $\mathrm{CO}$ recirculation from the effluent may be implemented to improve performance at the inlet region. Figure 6 represents the baseline case for the Nernst potential without recirculation. The open circuit voltage was calculated from a $\mathrm{CO}_{2}$ utilization rate of $1 \%$ so the equation is defined, to an approximated upper bound of the reaction at $50 \%$ utilization.

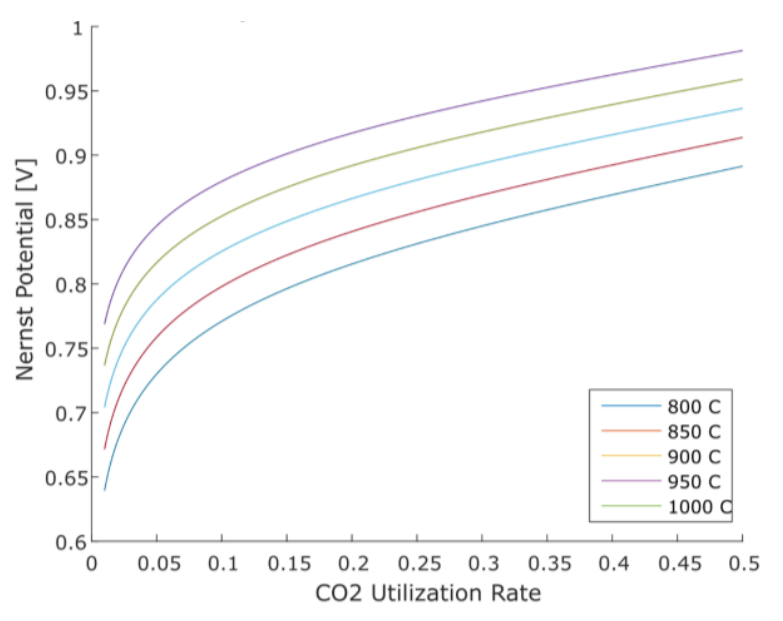

Figure 6 - Nernst potential as $\mathrm{CO}_{2}$ is consumed across the stack

In order to derive a better approximation of the Nernst potential for modeling the bulk properties of the SOXE as a component of the MOXIE system, a 2D approximation of the Nernst potential is derived. This approximation accounts for the high molar fraction of $\mathrm{CO}_{2}$ as it enters the cell, and proceeds to the targeted $50 \%$ utilization rate. This equation calculates the average Nernst potential over the length of the cell.

$$
\frac{1}{x_{c o, \text { in }}-x_{c o, \text { out }}} \int_{x_{c o, \text { in }}}^{x_{c c, o u t}} E_{0}+\frac{R T}{4 F} \ln \left[\frac{\left(P_{c} * x_{c o}\right)^{2} P_{O_{2}}^{0}}{\left(P_{c} *\left(1-x_{c o}\right)\right)^{2}}\right] d x_{c o}
$$

Where $x$ is the local $\mathrm{CO}_{2}$ utilization or the ratio of $\mathrm{CO}$ to $\mathrm{CO}_{2}$ in the stream, $P_{c}$ is the total pressure over the cathode. This captures Nernst potential changes longitudinally over the length of the cell as more $\mathrm{CO}_{2}$ is converted to $\mathrm{CO}$. The applied voltage is constant throughout the cell, and both potentials are unchanging laterally. $\mathrm{x}_{\mathrm{in}}$ is specified as $1 \%$ to avoid an undefined potential at the inlet stream. Integrating from a $\mathrm{CO}_{2}$ utilization rate of $1 \%$ to a rate $50 \%$ at $1073 \mathrm{~K}(800 \mathrm{C})$ and operating at 1 atmosphere yields an average Nernst potential of:

$$
V_{o c v}=0.936 \mathrm{~V}
$$

This is an average value for this particular case at our maximum estimated utilization rate. The Nernst potential just captures one component of the required potential to drive the reaction across the SOXE. The total required potential across the SOXE can be accounted for as

$$
V=V_{o c v}+\eta_{c o n c, c}+\eta_{a c t, c}+\eta_{a c t, a}+\eta_{\text {ohmic }}
$$

Where $\eta_{\text {conc,c }}$ is the cathode concentration overpotential, $\eta_{a c t, c}$ is the cathode activation potential loss, $\eta_{a c t, a}$ is the anode activation potential and $\eta_{\text {ohmic }}$ is the ohmic resistance potential loss. Several models have been developed to quantify some or all of these losses for solid oxide electrolysis (Sridhar and Vaniman 1997, Ni, Leung et al. 2006, Nehrir and Wang 2009, Ni 2010). These models require in-depth characterization of the SOXE parameters, and often borrow models from other fields that may or may not be valid for high 
temperature solid oxide electrolysis. Our model captures these values in the experimentally derived ASR value.

\section{G. Reaction conditions and heat generation.}

Now that we have established an approximation for open circuit voltage, we can now estimate the heat flow in and out of the stack based on the operating parameters. This section will establish the thermodynamic linkages between other parts of the system.

As discussed earlier, the thermal neutral voltage described in equation (14), is the voltage at which operating the reaction does not generate or consume heat. Above $V_{t n}$ the reaction is exothermic and below it is endothermic. The heat generated during the reaction per cell can be quantified by

$$
\dot{Q}_{g e n}=\left(V_{o p}-V_{t n}\right) * I
$$

If the stack is operated below the thermal neutral voltage, additional heat will need to be supplied to make up for the endothermic reaction. Operation at thermal neutral voltage requires some heat addition by the SOXE heaters to maintain steady operating temperature, as some heat is lost through the SOXE insulation.

\section{H. Relating SOXE Models to System Performance}

A major challenge of SOXE development and design is achieving a low ASR value. A low ASR is desirable because it allows a stack to have higher current (and therefore, production rate) with the same voltage. Figure 7 demonstrates the importance of ASR on SOXE stack performance for a giving cell voltage.

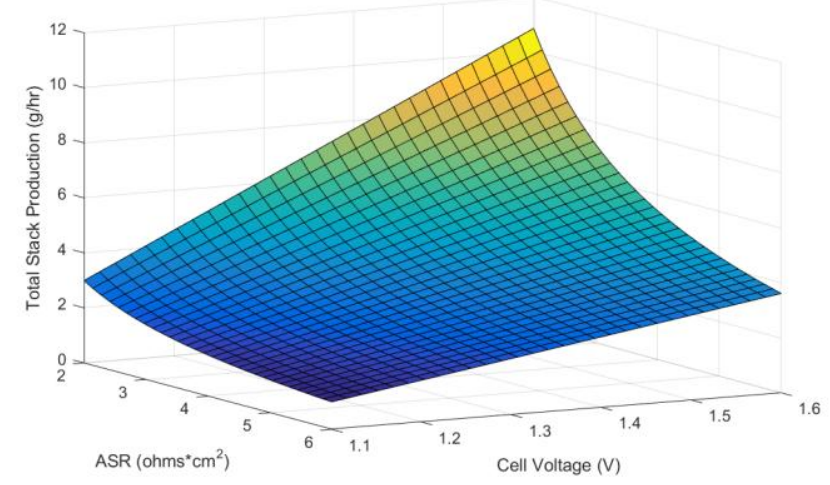

Figure 7 - Oxygen production rate and operating voltage for a 5 cell SOXE stack operating at $1073 \mathrm{~K}$.

High ASR cannot always be compensated for by applying a higher voltage across the stack. In solid oxide electrolysis of $\mathrm{CO}_{2}$, operating at high voltages increases the risk of a degradation process called coking. Coking is a process in which the cathode is fouled by carbon deposition and reduces the performance of the reaction. This risks increases and cell potential and utilization rates increase. One mechanism in which coke can form is the Boudouard reaction. (Bove and Ubertini 2008)

$$
2 \mathrm{CO}(g) \leftrightarrow \mathrm{CO}_{2}(g)+C(s)
$$

The coking threshold in the MOXIE SOXE cells has not yet been fully characterized. Early testing of the MOXIE stack has been conducted up to the thermal neutral voltage $\mathrm{V}_{\text {th }}=1.463$ V.

Future modeling and testing efforts will explore this limit.

\section{CONCLUSION AND FutURE WORK}

\section{A. Conclusion}

This paper describes the development of a thermodynamic model for solid oxide electrolysis, based primarily on first principles. All empirical inputs are incorporated in the term for area specific resistance (ASR), which is readily characterized during stack and cell testing.

Independent variables for this model include design parameters such as:

- Cell active area

- Number of cells

Operation parameters that are also independent variables include:

- Cell operating voltage

- Cell operating temperature

- Inlet stream flow rate and molar composition

- Outlet product stream pressure

- Estimated maximum utilization rate (can be iterative)

Dependent variables include:

- $\mathrm{O}_{2}$ production rate

- Stack and cell current

- Heat generated/consumed per cell

- $\mathrm{CO}_{2}$ utilization rate

Other key values that were derived include the temperature dependent properties of the thermal neutral voltage $\left(\mathrm{V}_{\mathrm{tn}}\right)$, the reversible voltage $\left(\mathrm{V}_{\text {rev }}\right)$, and the open circuit or Nernst potential $\left(\mathrm{V}_{\text {ocv }}\right)$.

This model establishes important relationships between the stack design, number of cells, operating temperature, operating voltage and oxygen production rate. The model links production rate, and flow to utilization rate, allowing for monitoring of desired parameters and high risk areas.

\section{B. Future work}

The next goal of the SOXE modeling is to incorporate a model of the potentials for a reaction that forms carbon coke. This model will help identify the theoretical thermodynamic limits of the electrolysis reaction before carbon dioxide is electrolyzed to carbon, damaging the electrode and reducing cell performance. This model will need to be tested in the lab to determine the appropriate margin of error to include accounting for non-ideal conditions. It has been shown that for $\mathrm{CO}_{2}$ electrolysis with an YSZ electrolyte that the actual coke formation can occur up to $5.4 \%$ before the theoretical Boudouard threshold (Skafte 2015). Such a model will help in establishing operation bounds for control system development and for optimizing the system performance.

An improved model of how the area specific resistance is affected by temperature, flowrate, and time dependent cell 
degradation will be developed to improve the overall model accuracy. Empirical relationships will be established with laboratory testing and enhanced with insights from theoretical modeling approaches.

Meanwhile, this model will be incorporated with the thermodynamic models of the other components of the system. This includes the scroll compressor, heaters, and viscous flow control devices that drop the pressure of the effluent gas to Mars ambient.

\section{ACKNOWLEDGEMENTS}

This work was supported by the National Science Foundation Graduate Research Fellowship Program and NASA. The author would also like to thank Dr. Gerald Voecks from NASA Jet Propulsion Laboratory and Dr. Joseph Hartvigsen from Ceramatec Inc. for their guidance.

\section{REFERENCES}

Bove, R. and S. Ubertini (2008). Modeling Solid Oxide Fuel Cells, Springer.

Drake, B. G. (2009). Human exploration of Mars: Design Reference Architecture 5.0. N. J. S. Center. Washington, D.C., NASA.

Ebbesen, S. D. and M. Mogensen (2009). "Electrolysis of carbon dioxide in solid oxide electrolysis cells." Journal of Power Sources 193(1): 349-358.

Hartvigsen, J., S. Elangovan, D. Larson, M. Elwell, M. Bokil, L. Frost and L. M. Clark (2015). Challenges of Solid Oxide Electrolysis for Production of Fuel and Oxygen from Mars Atmospheric CO2. ECS Conference on Electrochemical Energy Conversion \& Storage, Glasgow, Scotland, Ecs.

Nehrir, M. H. and C. Wang (2009). Modeling and control of fuel cells: distributed generation applications, John Wiley \& Sons.

$\mathrm{Ni}, \mathrm{M}$. (2010). "Modeling of a solid oxide electrolysis cell for carbon dioxide electrolysis." Chemical Engineering Journal 164(1): 246-254.

Ni, M., M. K. Leung and D. Y. Leung (2006). "A modeling study on concentration overpotentials of a reversible solid oxide fuel cell." Journal of Power Sources 163(1): 460-466.

Rapp, D., J. A. Hoffman, F. Meyen and M. H. Hecht (2015). The Mars Oxygen ISRU Experiment (MOXIE) on the Mars 2020 Rover. AIAA Space 2015 Conference and Exposition. Pasadena, California, AIAA. 2015-4561.

Senate", T. U. S. (2010). NASA Authorization Act of 2010. Washington, DC, USA.

Skafte, T. (2015). Carbon Deposition during CO 2 Electrolysis in Ni-Based Solid-Oxide-Cell Electrodes. ECS Conference on Electrochemical Energy Conversion \& Storage with SOFC-XIV (July 26-31, 2015), Ecs.

Sridhar, K. R. and B. T. Vaniman (1997). "Oxygen production on Mars using solid oxide electrolysis." Solid State Ionics 93(3-4): 321-328.

Stoots, C. M., J. E. O'Brien, J. S. Herring, K. G. Condie and J. J. Hartvigsen (2008). Idaho National Laboratory experimental research in high temperature electrolysis for hydrogen and syngas production. Fourth International Topical Meeting on High Temperature Reactor Technology, American Society of Mechanical Engineers.

Strickland, J. (2013). Revisiting SLS/Orion launch costs. The Space Review, The Space Review.
Williams, D. R. (2015). "Mars Fact Sheet." 2015, from http://nssdc.gsfc.nasa.gov/planetary/factsheet/marsfact.html. 